\title{
Auditory sensitivity after prolonged visual deprivation'
}

\begin{abstract}
Abstraet
Ss who were placed in darkness for a week but otherwise were exposed to a normal and varied sensory environment showed a significant increase in auditory flutter fusion frequency. This effect was still present one day after the termination of visual deprivation. The absolute threshold of hearing for five frequencies was not affected.
\end{abstract}

\section{Problem}

Recently it was reported that exposure of Ss to either a week of darkness or of unpatterned light produces a significant increase in tactual acuity and in sensitivity to heat and pain (Zubek et al, 1964a, b). This effect of visual deprivation was uniform. It was shown by almost all of the experimental Ss, on all skin areas, and on all cutaneous measures. Furthermore, the effects were still present several days after the termination of the experimental conditions. The purpose of this study is to determine whether visual deprivation can produce a similar increase in auditory sensitivity. If this should occur, it will suggest that prolonged visual deprivation may produce a general enhancement of sensory functioning.

\section{Method}

Fifteen male university students, each wearing a black mask, were placed in groups of two or three, in an ordinary room for a prescribed period of one week. Apart from the exposure to constant darkness, their environment was quite normal. No gloves were worn and no restrictions were placed on their motor activity or on conversation with one another or with the experimenters. Furthermore, a radio was available in the room at all times.

Two measures of auditory sensitivity, viz., auditory discrimination and absolute threshold of hearing, were taken before and after a week of darkness as well as at intervals of $1,2,5$, and 7 days after termination of visual deprivation. Auditory discrimination was determined by a fusion or flutter method. This measure, which is considered to be an auditory analogue of visual flicker (Miller \& Taylor, 1948) involves the production of an interrupted white noise whose frequency can be systematically increased until $\mathrm{S}$ reports a constant sensation of noise. The frequency at which this constant sensation occurs is usually referred to as the auditory flutter fusion frequency (AFF). The interrupted white noise, at a 0.90 on-off ratio and an intensity of approximately $70 \mathrm{db}$ re 0.0002 dynes $/ \mathrm{cm}^{2}$, was presented binaurally by a noise audiometer (Grason-Stadler,
Model 830-52). The method of limits, with descending series only, was used to measure the fusion threshold since it has been shown that a "minimal flutter stands out as a figure in a ground of repeated continuous noise bursts more distinctly than the reverse" (Symmes etal, 1955). Five practice and five experimental trial blocks, separated by a $1 \mathrm{~min}$. rest period, were administered at each testing session.

The second auditory measure consisted in the determination of the absolute threshold of hearing for five frequencies, viz., 100, 300, 1,000, 5,000 and 9,000 cps. The tones were generated by an audio-oscillator (Grason-Stadler, Model 950-D) and presented binaurally through Phillips earphones (Model HA-10). The maximum output obtained from the oscillator was $1.732 \mathrm{~V}$ with a 1,000 cps signal. This yielded a total acoustic output, binaurally, of $118.75 \mathrm{db}$ SPL re 0.0002 dynes $/ \mathrm{cm}^{2}$. The descending method of limits was used. Four blocks of trials were administered, each separated by a $1 \mathrm{~min}$. rest period. Each trial block contained the five test frequencies which were presented in a randomized manner. Practice trials were only given during the initial testing session. The two auditory tests were given in random order at each test session and the Ss were given $5 \mathrm{~min}$. rest periods between each measure to minimize fatigue effects. All tests were administered in a sound-proofed room. A group of 15 control Ss were given the same auditory measures and at the same time intervals as the experimental Ss. They were never visually deprived. Two-tailed t-tests for independent measures were used in the statistical analysis.

\section{Results}

Figure 1 indicates that the experimental Ss, after a week of visual deprivation, show a considerable increase in auditory flutter fusion frequency (greater resolving power) in relation to that of the control Ss $(p<.001)$. Fourteen of the 15 experimental Ss showed this increase. On the other hand, the control Ss exhibited a chance distribution of increases and decreases. Figure 1 also indicates that the effect was still present one day after termination of visual deprivation $(p<.01)$. The results on post-isolation days 2,5 , and 7 were not statistically significant. On the absolute threshold measures, both the experimental and control Ss showed a slight increase in sensitivity, on all five frequencies, after the one week interval. However, none of the "pre-post" differences between the two groups were statistically significant. Furthermore, no trend toward a greater sensitivity for the experimental Ss was evident. 


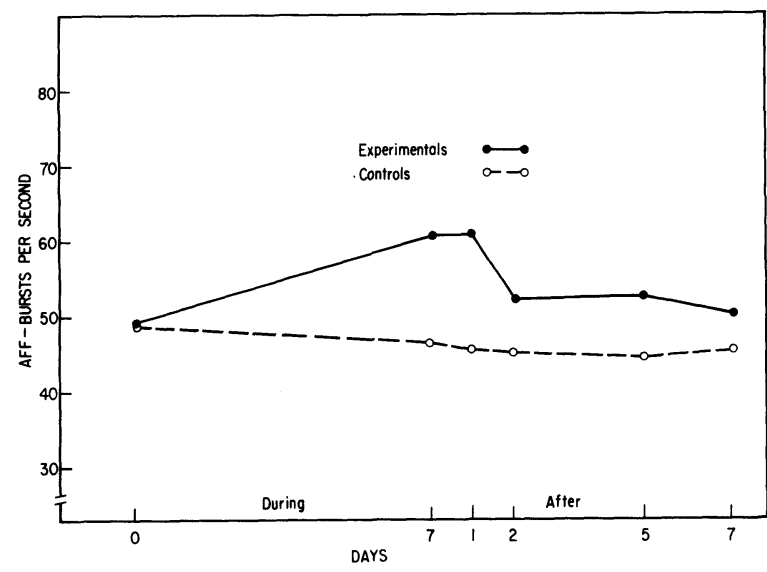

Fig. 1. Auditory flutter fusion frequency before and after a week of darkness, and 1, 2, 5, and 7 days later.

\section{Discussion}

The failure of the second auditory measure viz., absolute threshold of hearing, to show an increase in sensitivity is puzzling. It may, however, simply indicate that the facilitatory effects following prolonged visual deprivation apply only to sensory tests involving a temporal discrimination. Measures of absolute threshold may not be affected. This hypothesis is currently being tested. This apparent discrepancy in results may also be due to differences in auditory stimuli employed in the two types of tests. The absolute threshold measures used pure tones whereas the flutter fusion determinations employed a white noise. Some support for the importance of this variable was recently provided by Galin (1964) who demonstrated that white noise and pure tones produced qualitatively different patterns of evoked activity at different levels of the auditory system. The most notable difference occurred at the inferior colliculus where white noise produced a marked increase in activity while pure tonal stimulation had no such effect. This distinctiveness in physiological response to the two types of auditory stimuli may be an important factor in accounting for the differential effects on the two measures of auditory sensitivity.

The results of this experiment, together with our earlier research, indicate that prolonged visual deprivation has a facilitatory effect on both cutaneous and auditory discriminatory performance, an effect which persists for at least a day after restoration of normal visual stimulation. Furthermore, these results suggest that visual deprivation may produce a general enhancement of sensory functioning involving not only the cutaneous and auditory modalities but also smell, taste, and kinesthesis. Some support for this suggestion was recently provided by the Japanese investigator Nagatsuka (1965) who reported a $35 \%$ increase in taste sensitivity (sweet and bitter) following prolonged visual and auditory deprivation. Although two modalities were deprived, it is probable that a similar effect would result from visual deprivation alone.

Various lines of evidence suggest that these intersensory facilitatory effects may be mediated by the reticular activating system (RAS). First, this neural system receives afferent impulses from various sensory sources via collaterals of ascending tract fibers and transmits them diffusely to the cerebral cortex. Thus a mechanism for inter-sensory effects is present. Second, it has been suggested (Lindsley, 1961) that the RAS may act "as a sort of homeostat regulating or adjusting input-output relations.... . Its changes and adjustments depend upon the ebb and flow of activity in the afferent or efferent systems and when these are restricted, compensatory adjustments are made." One of these compensatory adjustments may be a facilitation of certain sensory processes. Third, facilitation of sensory discrimination can occur following electrical stimulation of the RAS. For example, Fuster (1958) has shown that stimulation of the brain-stem reticular system of monkeys, while they were engaged in the performance of visual discrimination tasks, increased their speed of reaction, improved their discriminatory accuracy, and lowered their tachistoscopic recognition thresholds. Lindsley (1961) has also reported that stimulation of the RAS "improved the resolving power of efficiency" in the visual cortex to two brief flashes of light.

\section{References}

Fuster, J. M. Effects of stimulation of brain stem on tachistoscopic perception. Science, 1958, 127, 150.

Galin, D. Auditory nuclei: distinctive response patterns to white noise and tones in unanesthetized cats. Science, 1964, 146, 270-272

Lindsley, D. B. Common factors in sensory deprivation, sensory distortion, and sensory overload. In P. Solomon et al (Eds.), Sensory deprivation. Cambridge: Harvard University Press, 1961. Pp. 174-194

Miller, G. A., \& Taylor, W. G. The perception of repeated bursts of noise. J. Acoust. Soc. Amer., 1948, 20, 171-182.

Nagatsuka, Y. Studies on sensory deprivation: III. Part 2. Effects of sensory deprivation upon perceptual functions. Tohoku Psychol. Folia, 1965, 23, 56-59.

Symmes, D., Chapman, L. F., \& Halstead, W. C. The fusion of intermittent white noise. J. Acoust. Soc. Amer., 1955, 27, 470473.

Zubek, J. P., Flye, J., \& Aftanas, M. Cutaneous sensitivity after prolonged visual deprivation. Science, 1964a, 144, 1591-1593.

Zubek, J. P., Flye, J., \& Willows, D. Changes in cutaneous sensitivity after prolonged exposure to unpatterned light. Psychon. Sci., 1964b, 1, 283-284.

\section{Note}

1. This research was supported by the National Research Council, Canada (APT-106) and the Defence Research Board, Canada (No. 9425-08). Appreciation is expressed to Dr. D. S. Abbey for assistance at various stages of the project. 